\title{
TLR4 Inhibitor Alleviates Sepsis-Induced Organ Failure by Inhibiting Platelet Mtros Production, Autophagy, and Gpiib/liia Expression
}

\section{Ying Li}

Central South University Third Xiangya Hospital

Guo Feng ( $\nabla$ medfg@outlook.com)

Central South University Third Xiangya Hospital https://orcid.org/0000-0001-5177-8101

\section{Research Article}

Keywords: Toll Like Receptor 4 inhibitor, Sepsis, Platelet, Mitochondrial ROS, Autophagy

Posted Date: December 17th, 2021

DOI: https://doi.org/10.21203/rs.3.rs-1156638/v1

License: (c) (i) This work is licensed under a Creative Commons Attribution 4.0 International License.

Read Full License 


\section{Abstract}

Thrombocytopenia and impaired platelet aggregation are associated with sepsis-induced organ failure. Many studies have shown that mitochondrial ROS, autophagy is related to organ injury in sepsis. However, the relationship between them is unknown. Here, we explored whether Toll Like Receptor 4 inhibitor alleviates sepsis organ failure by inhibiting platelet mitochondrial ROS production, autophagy, and GPIlb/Illa expression. We found Toll Like Receptor 4 inhibitor TAK242 alleviated LPS-induced acute kidney and lung injury and decrease platelet activation in a mouse model of sepsis mice. In vitro study, inhibiting Toll Like Receptor 4 reduced inward flow of $\mathrm{Ca}^{2+}$ and decreased endogenous mitochondrial ROS production in platelets treated with LPS, and TAK242 inhibited autophagy and NOX expression in LPS treated platelets. Thus, we supposed that Toll Like Receptor 4 inhibitor effectively alleviate lung and kidney injury in a mouse model of sepsis induced by LPS and its effects are related to the inhibition of mouse platelets GPIlb/IIla, and also can reduce LPS-induced mitochondrial ROS generation related to $\mathrm{Ca}^{2+}$ influx, thus reducing platelet activation. LPS can induce platelet autophagy by generating mitochondrial ROS, which may be the pathophysiological mechanism of organ injury in sepsis.

\section{Introduction}

Sepsis is a critical illness with extremely high mortality in intensive care units (ICUs), with an incidence of $6-30 \%$ [1]. The mortality from sepsis is as high as $28 \%-50 \%$ because of multiple organ failure such as sepsis-induced lung injury and kidney failure[2].A study from over 40 years ago found that sepsis is closely related to thrombocytopenia[3]. Platelet activation and consumption due to inflammatory storm and a platelet count less than $50,000 / \mu \mathrm{L}$ are indicators of poor prognosis in sepsis patients $[4,5]$. Thrombocytopenia and Iimpaired platelet aggregation are associated with sepsis-related mortality and sepsis-induced organ failure. Disorders of the coagulation mechanism, microthrombosis, vascular obstruction, and insufficient perfusion of tissues and organs caused by abnormal platelet function are the pathophysiological basis of organ dysfunction. Increased activation of the platelet surface marker sPselectin is related to acute lung injury[6-11] and platelet aggregation leads to acute kidney injury by promoting microvesicle concentration [12]区

Intracellular Reactive Oxygen Species (ROS) induced by bacterial liposaccharide (LPS) through Toll Like Receptor (TLR4) can induce mitochondrial damage and activate platelets. However, the source of intracellular ROS and related molecular mechanisms have not been shown[13]. Mitochondria are involved in pathophysiological processes such as energy production, intracellular signal transduction and cell death regulation, and play a key role in cell metabolism, survival and homeostasis. Many studies have shown that mitochondrial disorders are related to impaired organ function in sepsis.

ROS mainly come from the Nicotinamide Adenine Dinucleotide Phosphate (NADPH) pathway and the mitochondrial electron transporter pathway. Organ failure in sepsis is mainly related to the increase of mtROS. Reducing the production of mtROS can alleviate lung injury in sepsis[14]. Moreover, autophagy may be upstream of the production of mtROS, and is related to organ injury in sepsis. LPS can induce 
autophagy and mtROS production in macrophages to induce acute liver injury[15]. Inhibiting autophagy in macrophages can inhibit the generation of mtROS and reduce inflammation[16]. Autophagy is related to septic kidney injury induced by LPS via the PI3K/AKT/mTOR pathway, and inhibition of autophagy can reduce septic kidney injury [17].

Despite lacking a nucleus, platelets have rich internal organelles such as mitochondria and lysosomes, may be a key target for the regulation of micro-thrombosis and inflammation in sepsis. According to current research, LPS causes the release of mtROS through TLR4, leading to impaired platelet function, which is significant for organ dysfunction in sepsis. However, the signal pathways through which platelet TLR4 is activated to mediate organ injury in sepsis are still unclear. In previous in vivo studies, our team found that the use of TAK242 to inhibit the expression of platelet TLR4 can reverse LPS-mediated septic organ injury in mice. Here, we explored whether TLR4 inhibitor alleviates sepsis organ failure by inhibiting platelet mtROS production, autophagy, and GPIIb/IIla expression.

\section{Methods And Materials}

\subsection{Preparation of platelets}

Peripheral venous blood of healthy volunteers and Blood collected from mice heart treated with anticoagulant then the blood was centrifuged for $10 \mathrm{~min}$ at $150 \mathrm{~g}$, and then the PRPs was collected. PRPs were washed with $\mathrm{Ca}^{2+}$ free-Tyrodes HEPES buffer and then centrifuged for $10 \mathrm{~min}$ at $850 \mathrm{~g}$. The supernatant was discarded and then washed platelets was suspended in $\mathrm{Ca}^{2+}$ Free-Tyrodes HEPES buffer.

\subsection{Materials}

LPS was purchased from Sigma Chemicals (St. Louis, MO, USA). TAK242 (TLR4 receptor inhibitor) was purchased from MedChem Express (New Jersey, USA). $\mathrm{H}_{2} \mathrm{O}_{2}$ was purchased from Sinopharm Chemical Reagent Company (Shanghai, China). N-acetyl-L-cysteine (NAC) and Calcium Detection were bought from Byotime (Shanghai, China). GAPDH, RIPA buffer, PMSF, protease, and phosphatase inhibitor cocktails were obtained from Cell Signaling Technology (Beverly, MA, USA). MitoSOX ${ }^{\mathrm{TM}}$ Red mitochondrial superoxide indicator (MitoSOX ${ }^{\mathrm{T} M}$ ) was obtained from Thermo Fisher (USA).

\subsection{Flow cytometric analysis}

PRPs $\left(2 \times 10^{7} / \mathrm{mL}\right)$ were separately treated with LPS $(10 \mu \mathrm{g} / \mathrm{ml})$, LPS $(10 \mu \mathrm{g} / \mathrm{ml})$ and TAK242 $(132 \mathrm{nM})$, NAC $(5 \mathrm{mM}), \mathrm{H}_{2} \mathrm{O}_{2}(100 \mu \mathrm{M})$, or vehicle at $37^{\circ} \mathrm{C}$ for $30 \mathrm{~min}$. We diluted the $5 \mathrm{mM}$ MitoSOX ${ }^{\mathrm{TM}}$ reagent stock solution in $\mathrm{HBSS} / \mathrm{Ca} / \mathrm{Mg}$ to make a $5 \mu \mathrm{M}$ MitoSOX ${ }^{\mathrm{TM}}$ reagent working solution. .We applied $1.0-2.0 \mathrm{~mL}$ of $5 \mu \mathrm{M}$ MitoSOX ${ }^{\mathrm{TM}}$ reagent working solution to cover PRPs adhering to coverslip(s). We then incubated cells for $10 \mathrm{~min}$ at $37^{\circ} \mathrm{C}$, protected from light. We incubated the calcium probe with PRPs for 15 minutes in a thermostatic dark box at $37^{\circ} \mathrm{C}$ and then analysis the fluorescence intensity by a flow cytometer (BD Calibur, USA). 


\subsection{Western blot analysis}

Washed platelet lysates $\left(10^{9}\right.$ cells $\left./ \mathrm{mL}\right)$ were incubated with LPS $(10 \mu \mathrm{g} / \mathrm{ml}), \mathrm{LPS}(10 \mu \mathrm{g} / \mathrm{ml})$ combined with TAK242(132 nM) or vehicle for 5 min at $37^{\circ} \mathrm{C}$. Processed samples were lysed and equal-amounts of protein were subjected to $12 \%$ SDS-PAGE and transferred onto PVDF for subsequent probing (LC3B or human NOX2).

\subsection{Sepsis organ injury and the effect of TLR4 inhibitor in vivo.}

Male BALB/c mice (20-25 g) were purchased from SLAC Laboratory Animal Central (Changsha, China). Animals are bred in an air-conditioned room with temperature of $25^{\circ} \mathrm{C}$ and relative humidity of $55 \%$, and acclimated for 7 days under a regular light-dark cycle. Mice in the LPS group received dorsal subcutaneous injections of LPS $(0.18 \mu \mathrm{g} / \mathrm{ml})$ from day 2 to day 3 . Mice in the TLR4 inhibited group received dorsal subcutaneous injections of TAK242 $(5 \mathrm{mg} / \mathrm{kg})$ from the first day to the third day, and LPS $(0.18 \mu \mathrm{g} / \mathrm{ml})$ was used on day 2 to day 3 . Mice in the vehicle group received subcutaneous injection of saline solution with equivalent volume on days 1-3. After sacrificed, mice lungs and kidneys were harvested for HE staining and photographed at 200x and 400x, respectively. GPRPs was separated from heart whole blood and was analyzed by flow cytometric analysis.

\section{Results}

\subsection{TLR4 inhibitor alleviates LPS-induced acute kidney and lung injury and decreases platelet activation in a mouse model of sepsis mice.}

To investigate the effect of LPS and TLR4 inhibitor on mice, histopathological studies were performed. H\&E staining showed that LPS injection caused acute kidney and lung injury. LPS induced edema of renal tubular epithelial cells and hemorrhage (Fig. 1A). LPS induced increased pulmonary vascular permeability and pulmonary hemorrhage compared with the control group (Fig. 1B). TAK242 (20 mg/kg) efficiently prevented both kidney and lung injury and inflammatory cell infiltration into kidney and lung tissue, as shown by H\&E staining compared with LPS group.

Blood samples were collected from the three groups of mice and the Platelet Rich Plasma (PRPs)were separated. The proportion of CD41(+)CD62P(-) platelets in mice treated with LPS was increased compared with that of the blank control group $(\mathrm{p}<0.05)$. The proportions of CD41(-) CD62P(+) and $\mathrm{CD} 41(+) \mathrm{CD} 62 \mathrm{P}(+)$ platelets were significantly higher than that of the blank control group $(p<0.001)$, and the proportion of CD41(-)CD62P(-) platelets was slightly lower than that of the blank control group $(p<0.01)$. The proportion of CD41(+) CD62P(-) platelets in the TAK242 intervention group was significantly lower than that in the LPS group $(p<0.01)$, and there was no statistical difference compared with the blank group ( $p>0.05)$. The proportion of $\operatorname{CD} 41(-) \operatorname{CD} 62 \mathrm{P}(+)$ and $\operatorname{CD} 41(+) \operatorname{CD} 62 \mathrm{P}(+)$ platelets was 
significantly lower in the TAK242 intervention group than that of the LPS control group, and the proportion of CD41(-) CD62P(-) platelets was slightly higher than that of the LPS group and not statistically different than that of the blank group ( $p>0.05)$ (Fig. 1C).

\subsection{Inhibiting TLR4 reduces inward flow of $\mathrm{Ca}^{2+}$ in platelets treated with LPS}

In previous study, we found that LPS induced an increase in the expression of P-selectin on the surface of platelets and an increase in the maximum platelet aggregation rate via releasing endogenous ROS and ultimately leading to platelet activation[13]. Platelet activation is related to the influx of calcium ions[18]. Here, we used the calcium ion probe Fluo-3 AM ester which penetrates the platelet cell membrane and combines with intracellular calcium ions to generate strong fluorescence. We found that the calcium ion concentration in platelets treated with LPS increased significantly, (Fig. 2, LPS v.s. NC, $P<0.001$ ), but

found no difference between the LPS and $\mathrm{H}_{2} \mathrm{O}_{2}$ groups (Fig. 2, LPS v.s. $\mathrm{H}_{2} \mathrm{O}_{2}, \mathrm{P}>0.05$ ). The TLR4 inhibitor TAK242 decreased the intraplatelet calcium ion concentration after intervention by LPS (Fig. 2, LPS+TAK242 v.s. LPS, P<0.001) to levels that were indistinguishable from those of the blank group (Fig. 2 , LPS+TAK242 v.s. NC, $P>0.05$ ). No statistically significant difference was found between the effects of TAK242 and the antioxidant drug NAC (Fig. 2, LPS+TAK242 v.s. LPS+NAC, P>0.05).

\subsection{TLR4 inhibitors decreased endogenous mtROS production in LPS-induced platelets}

In previous study, we found that LPS promoted the release of intracellular ROS through platelet TLR4 to activate platelets. In this study, we found that LPS can induce platelets to produce large amounts of mtROS. (Fig. 3, LPS v.s. NC, P<0.0001), TLR4 inhibitors can decrease mtROS production by LPS-induced platelets (Fig. 3, LPS+TAK242 v.s. LPS, P<0.001), and the inhibitory effect of TLR4 inhibitor on platelet mtROS is no different from that of the oxygen free radical scavenger NAC (Fig. 3, LPS+NAC v.s. LPS+TAK242, $P>0.05$ ). The effect of $\mathrm{H}_{2} \mathrm{O}_{2}$ on platelet aggregation in the positive control group was not different from that of the LPS group (Fig. $3, \mathrm{H}_{2} \mathrm{O}_{2}$ v.s. LPS, $\mathrm{P}>0.05$ ).

\subsection{TAK242 inhibited autophagy and NOX expression in LPS treated platelets}

LC3B is widely used as an indicator of autophagy. The ratio of LC3BII and LC3BI can be used to estimate autophagic flux. In this study, we found that LPS induces autophagic flux in platelets using Western blot analysis of LC3BII and LC3BI (Fig. 4, LPS v.s. NC, P<0.01). We also found that TLR4 inhibitors reduce LPS-induced autophagy (Fig. 4, LPS+TAK242 v.s. LPS, $P<0.01$ ).

Nicotinamide adenine dinucleotide (phosphate) $(\mathrm{NAD}(\mathrm{P}) \mathrm{H})$ oxidase $(\mathrm{NOX})$ isoforms are the main sources of ROS in platelets, followed by cyclooxygenase (COX), xanthine oxidase (XO), and mitochondrial respiration[19]. We found that LPS induced a significant increase in platelet NOX expression (Fig. 4, LPS 
v.s. NC, P<0.01) and that the TLR4 inhibitor TAK242 decreased the expression of NOX in LPS-treated platelets (Fig. 4, LPS+TAK242 v.s. LPS, P<0.05). However, the expression of NOX in LPS+TAK242 group was still slightly higher than that in the blank group (Fig. 4, LPS+TAK242 v.s. NC, P<0.05).

\section{Discussion}

Platelets are particles shed from the cytoplasm during the maturation of megakaryocytes. They are rich in organelles and contents but with no nucleus. The process of platelet activation is mainly completed by these organelles and their contents. Platelet activation is accompanied by the expression of many specific surface markers, such as platelet granule membrane glycoprotein P-selectin (CD62P) and platelet plasma membrane glycoprotein (CD41 or GPIlb)[20]. During the resting period, the platelet membrane surface contains 800-1120 CD62P molecules per platelet on average. When platelets are activated by thrombin, the alpha granules in the platelet fuse with the plasma membrane through the Open Canalicular System (OCS), increasing the number to $1.0-1.3 \times 10^{3} \mathrm{CD} 62 \mathrm{P}$ molecules on the plasma membrane.

Many bacterial proteins directly promote the expression of the GPIIb/IIla complex via binding to the arginine-glycine-aspartic acid (RGD) sequence on the platelet surface, and thus activate the platelet [21]. Therefore, in this study, we used the platelet activation markers CD41, representing GPIIb/Illa, and CD62P, representing PS ectropion. We successfully constructed a mouse model of sepsis by subcutaneous injection of LPS. We observed sepsis-induced pulmonary hemorrhage and kidney injury in mice by HE staining of pathological sections. The platelet-rich plasma of septic mice showed an increase in the expression of platelet-activated surface antibodies. The expression of CD41 increased more than the expression of CD62P. This might be related to the fact that flow cytometry results cannot accurately measure the precise number of specific glycoproteins on the surface of each platelet. Our results are consistent with prior work[21].

LPS binds to its receptor TLR4 on the surface of platelets to mediate micro thrombosis and organ injury due to insufficient perfusion. Furthermore, in response to Escherichia coli infection, platelet TLR4 contributes to bacterial trapping by supporting NET formation[22]. Platelets promote leukocyte recruitment in LPS-induced lung injury. In contrast, low platelet counts are associated with increased secondary hemostasis, kidney damage, and exacerbated bacteremia and systemic bacterial dissemination in bacteria induced sepsis [23-25]. There are many ways to produce intracellular ROS in cells. Bacterial invasion transfers the TNF receptor associated factor 6 (TRAF6) to the outer mitochondrial membrane through TLR4, and regulates the process of oxidative phosphorylation via Evolutionarily Conserved Signaling Intermediate in Toll Pathways (ECSIT), which ultimately leads to the ubiquitination of TRAF6-dependent ECSIT and the generation of mtROS [26]. In several model systems, $\mathrm{Ca} 2+$ concentration in blood is significantly lower when sepsis occurs, while Ca2+ overload appears in mitochondria [27-29]. Ca2+ overload acts as a signal to open Mitochondrial permeability transition pore (MPTP) in sepsis internal environment simulation experiments[30]. 
Based on our previous research, we further found that LPS stimulated Ca2+ influx in platelets. The effect of LPS on $\mathrm{Ca} 2+$ influx in platelets is consistent with the effect of adding exogenous reactive oxygen species (i.e. $\mathrm{H}_{2} \mathrm{O}_{2}$ ). The inhibitory effect of TLR4 inhibitor TAK242 on LPS-induced $\mathrm{Ca} 2+$ in platelets is consistent with the effects of the ROS scavenger NAC. This phenomenon is analogous to the effect of LPS on platelet-induced intracellular ROS production described in our previous study.

Mitochondria-targeted hydroethidine (Mito-SOX) is a redox probe in which the HE molecule is conjugated to a triphenyl phosphonium group (TPPp) via an alkyl side chain. Mito-SOX is targeted to mitochondria because of the presence of the TPPp moiety[31]. It has excitation/emission maxima of approximately $510 / 580 \mathrm{~nm}$. We therefore used the fluorescence intensity detected in the PE channel of the flow cytometer to determine mtROS levels in platelets.

Exogenous $\mathrm{H}_{2} \mathrm{O}_{2}$ can induce endothelial cell mitochondria to produce mtROS (32). We therefore used $\mathrm{H}_{2} \mathrm{O}_{2}$ as a positive control[32]. We found that $\mathrm{H}_{2} \mathrm{O}_{2}$ in mitochondria induces the production of a large amount of mtROS. LPS induced production pf a large amount of mtROS on platelets, exhibiting the same effect as $\mathrm{H}_{2} \mathrm{O}_{2}$. The inhibitory effect of TAK242 of mtROS production is not significantly different from that of the LPS combined with NAC group, suggesting that TAK242 and NAC have the same effect on mtROS production induced by LPS. Meanwhile, in vivo experiments showed that TAK242 inhibited the aggregation of platelets in mice and reduced lung and kidney injury in septic mice. We speculate that TLR4 inhibitors can reduce platelet activation by inhibiting platelet mtROS to alleviate organ injury in sepsis. We further conclude that intracellular ROS of platelets activated by LPS in our previous study were likely mtROS.

mtROS are incorporated into signaling pathways, including those regulating immune responses and autophagy. NOX isoforms are the main sources of ROS in platelets, followed by cyclooxygenase (COX), xanthine oxidase (XO), and mitochondrial respiration[19]. mtROS and NOX-dependent ROS come from different pathways but are closely connected. In neutrophils and endothelial cells, as the concentration of $\mathrm{mtROS}$ and NOX-derived ROS in the cytoplasm increases, activation of PKC and $\mathrm{CcSrc}$ by mtROS upon its translocation to the cytosol leads to ROS production by NOX2[33, 34]. In our previous study, we found that LPS decreases p-PKC expression in platelets, but in this study, we found that LPS increases NOX2 expression in platelets and a concomitant increase in mtROS. The inhibition of PKC expression may be related to the increase of endogenous mtROS[35].

mtROS are closely related to autophagy. In physiological conditions, autophagy helps cells remove damaged mitochondria and peroxidized proteins. In most cases, autophagy supports survival. Intracellular ROS cause mitochondrial damage, thereby initiating autophagy to clear damaged mitochondria[13]. Therefore, autophagy has a protective effect against organ damage in sepsis. Blocking liver autophagy in a murine model of sepsis accelerates hepatocyte apoptosis and mitochondrial damage and decreases survival[36]. Interestingly, it was proposed in 2015 that platelet activation can induce autophagy in platelets and is closely related to thrombocytopenia and thrombosis [37]. Consistent 
with this hypothesis, we found that LPS induces platelet aggregation through release of mtROS, accompanied by platelet autophagy.

\section{Conclusion}

5.1 TLR4 inhibitors effectively alleviate lung and kidney injury in a mouse model of sepsis induced by LPS and its effects are related to the inhibition of mouse platelets GPIIb/IIla.

5.2 TLR4 inhibitors can reduce LPS-induced mtROS generation related to $\mathrm{Ca}^{2+}$ influx, thus reducing platelet activation.

5.3 LPS can induce platelet autophagy by generating mtROS, which may be the pathophysiological mechanism of organ injury in sepsis.

\section{Declarations}

\section{Ethics approval and consent to participate}

Volunteers were aware of this study and signed an informed consent form. All procedures were conducted in accordance with the protocol approved by the ethical committee of the Laboratory Animals Center of Central South University and all the procedures were under the guideline of the Third Xiangya Hospital of Central South University Human Research Committee.

\section{Author contributions}

Guo Feng designed this study. Guo Feng and Ying Li performed the research, analyzed data, and wrote the paper. All authors read and approved the final version of this manuscript.

\section{Funding}

This research did not receive any specific grant from funding agencies in the public, commercial, or notfor-profit sectors.

\section{Data Availability}

The datasets generated during and analysed during the current study are not publicly available due to privacy of volunteers are involved but are available from the corresponding author on reasonable request.

\section{Informed Consent}


The authors affirm that human research participants provided informed consent for publication of the images in Figure(s) 1a, 1b and Figure(s)2-4.

\section{References}

1. Martin GS (2012) Sepsis, severe sepsis and septic shock: changes in incidence, pathogens and outcomes. Expert Rev Anti Infect Ther 10(6):701-706

2. Martin GS et al (2003) The epidemiology of sepsis in the United States from 1979 through 2000. N Engl J Med 348(16):1546-1554

3. Bone RC, Francis PB, Pierce AK (1976) Intravascular coagulation associated with the adult respiratory distress syndrome. Am J Med 61(5):585-589

4. Thiery-Antier N et al (2016) Is Thrombocytopenia an Early Prognostic Marker in Septic Shock? Crit Care Med 44(4):764-772

5. Claushuis TA et al (2016) Thrombocytopenia is associated with a dysregulated host response in critically ill sepsis patients. Blood 127(24):3062-3072

6. Baughman RP et al (1993) Thrombocytopenia in the intensive care unit. Chest 104(4):1243-1247

7. Lundahl TH et al (1998) Impaired platelet function correlates with multi-organ dysfunction. A study of patients with sepsis. Platelets 9(3-4):223-225

8. Adamzik $\mathrm{M}$ et al (2012) Whole blood impedance aggregometry as a biomarker for the diagnosis and prognosis of severe sepsis. Crit Care 16(5):R204

9. Sakamaki F et al (1995) Soluble form of P-selectin in plasma is elevated in acute lung injury. Am J Respir Crit Care Med 151(6):1821-1826

10. Russwurm S et al (2002) Platelet and leukocyte activation correlate with the severity of septic organ dysfunction. Shock 17(4):263-268

11. Gawaz M et al (1997) Platelet function in septic multiple organ dysfunction syndrome. Intensive Care Med 23(4):379-385

12. Zafrani $L$ et al (2012) Calpastatin controls polymicrobial sepsis by limiting procoagulant microparticle release. Am J Respir Crit Care Med 185(7):744-755

13. Feng $\mathrm{G}$ et al (2018) LPS enhances platelets aggregation via TLR4, which is related to mitochondria damage caused by intracellular ROS, but not extracellular ROS. Cell Immunol 328:86-92

14. Wang $\mathrm{H}$ et al (2021) The mitochondrial redistribution of eNOS is involved in lipopolysaccharide induced inflammasome activation during acute lung injury. Redox Biol 41:101878

15. Unuma $\mathrm{K}$ et al (2015) Extrusion of mitochondrial contents from lipopolysaccharide-stimulated cells: Involvement of autophagy. Autophagy 11(9):1520-1536

16. Fei Q et al (2020) Metformin protects against ischaemic myocardial injury by alleviating autophagyROS-NLRP3-mediated inflammatory response in macrophages. J Mol Cell Cardiol 145:1-13 
17. Zhao Y et al (2020) Dexmedetomidine Protects Against Lipopolysaccharide-Induced Acute Kidney Injury by Enhancing Autophagy Through Inhibition of the PI3K/AKT/mTOR Pathway. Front Pharmacol 11:128

18. Shiraishi M, Tani E, Miyamoto A (2010) Modulation of rabbit platelet aggregation and calcium mobilization by platelet cholesterol content. J Vet Med Sci 72(3):285-292

19. Wachowicz B et al (2002) Generation of reactive oxygen species in blood platelets. Platelets 13(3):175-182

20. Metzelaar MJ et al (1992) Identification of a 33-Kd protein associated with the alpha-granule membrane (GMP-33) that is expressed on the surface of activated platelets. Blood 79(2):372-379

21. Brennan MP et al (2009) Elucidating the role of Staphylococcus epidermidis serine-aspartate repeat protein G in platelet activation. J Thromb Haemost 7(8):1364-1372

22. Stark RJ, Aghakasiri N, Rumbaut RE (2012) Platelet-derived Toll-like receptor 4 (TIr-4) is sufficient to promote microvascular thrombosis in endotoxemia. PLoS ONE 7(7):e41254

23. Xiang B et al (2013) Platelets protect from septic shock by inhibiting macrophage-dependent inflammation via the cyclooxygenase 1 signalling pathway. Nat Commun 4:2657

24. de Stoppelaar SF et al (2014) Thrombocytopenia impairs host defense in gram-negative pneumoniaderived sepsis in mice. Blood 124(25):3781-3790

25. Powers ME et al (2015) Synergistic Action of Staphylococcus aureus a-Toxin on Platelets and Myeloid Lineage Cells Contributes to Lethal Sepsis. Cell Host Microbe 17(6):775-787

26. Vogel RO et al (2007) Cytosolic signaling protein Ecsit also localizes to mitochondria where it interacts with chaperone NDUFAF1 and functions in complex I assembly. Genes Dev 21(5):615-624

27. Bouillot S, Reboud E, Huber P (2018) Functional Consequences of Calcium Influx Promoted by Bacterial Pore-Forming Toxins.Toxins (Basel), 10(10)

28. Stelzner K et al (2020) Intracellular Staphylococcus aureus Perturbs the Host Cell Ca(2+) Homeostasis To Promote Cell Death.mBio, 11(6)

29. He W et al (2020) Hypocalcemia in sepsis: analysis of the subcellular distribution of $\mathrm{Ca} 2+$ in septic rats and LPS/TNF-a-treated HUVECs. J Infect Dev Ctries 14(8):908-917

30. Fedotcheva N, Olenin A, Beloborodova N (2021) Influence of Microbial Metabolites on the Nonspecific Permeability of Mitochondrial Membranes under Conditions of Acidosis and Loading with Calcium and Iron lons.Biomedicines, 9(5)

31. Zielonka J et al (2008) Cytochrome c-mediated oxidation of hydroethidine and mito-hydroethidine in mitochondria: identification of homo- and heterodimers. Free Radic Biol Med 44(5):835-846

32. Kim YM et al (2017) ROS-induced ROS release orchestrated by Nox4, Nox2, and mitochondria in VEGF signaling and angiogenesis. Am J Physiol Cell Physiol 312(6):C749-c764

33. Dikalov SI et al (2014) Nox2-induced production of mitochondrial superoxide in angiotensin IImediated endothelial oxidative stress and hypertension. Antioxid Redox Signal 20(2):281-294 
34. Kröller-Schön S et al (2014) Molecular mechanisms of the crosstalk between mitochondria and NADPH oxidase through reactive oxygen species-studies in white blood cells and in animal models. Antioxid Redox Signal 20(2):247-266

35. Song XM et al (2017) Aldose reductase inhibitors attenuate $\beta$-amyloid-induced TNF-a production in microlgia via ROS-PKC-mediated NF-KB and MAPK pathways. Int Immunopharmacol 50:30-37

36. Oami T et al (2018) Blocking Liver Autophagy Accelerates Apoptosis and Mitochondrial Injury in Hepatocytes and Reduces Time to Mortality in a Murine Sepsis Model. Shock 50(4):427-434

37. Ouseph MM et al (2015) Autophagy is induced upon platelet activation and is essential for hemostasis and thrombosis. Blood 126(10):1224-1233

\section{Figures}


A

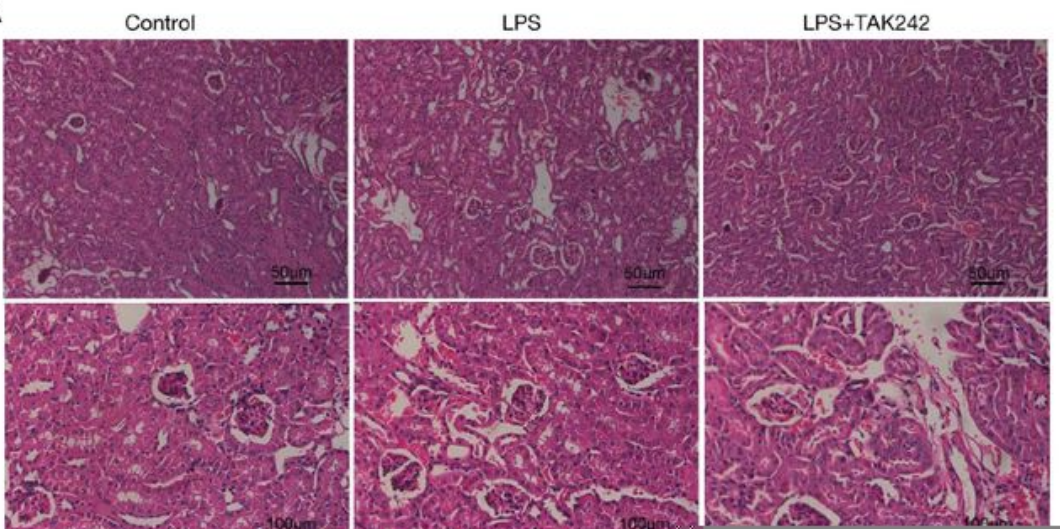

Figure 1

TAK242 improves LPS-mediated sepsis-induced lung and kidney injury. A. Under an optical microscope, H\&E-stained mouse kidney tissue was visualized at 200x and 400x magnification after treatment with saline, LPS, or LPS combined with TAK242, $n=3$ in each group. B. Under an optical microscope, H\&Estained mouse lung tissue was visualized at $200 \times$ and $400 \times$ magnification after treatment with saline, LPS, or LPS combined with TAK242, $n=3$ in each group. C. CD41 and CD62p levels on the surface of 
platelets measured by flow cytometry. Measurement data are represented as mean $\pm S E M,{ }^{*} p<.05,{ }^{*} p<$ $.01, * \star \star p<.001, * \star \star \star x p<.0001$.)
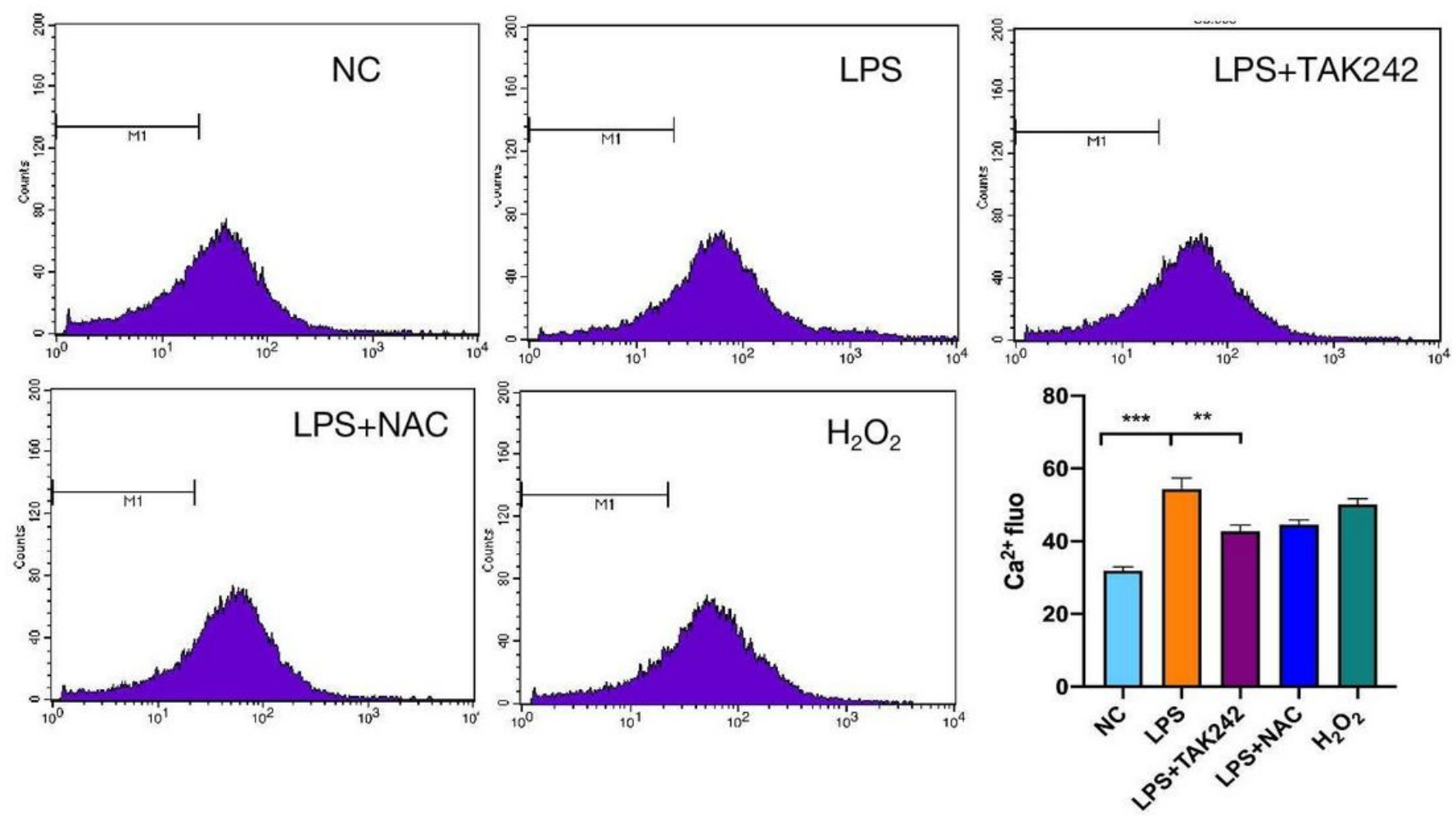

Figure 2

TAK242 reduced calcium influx induced by LPS in platelets. The intercellular Ca2+ concentration ([Ca2+]i) production was assayed by flow cytometry, $n=3$ in each group. The histogram displayed the MFI of $[\mathrm{Ca} 2+]$ i. Measurement data are represented as mean $\pm \mathrm{SEM},{ }^{\star \star} \mathrm{p}<.01,{ }^{\star \star *} \mathrm{p}<.001$. 

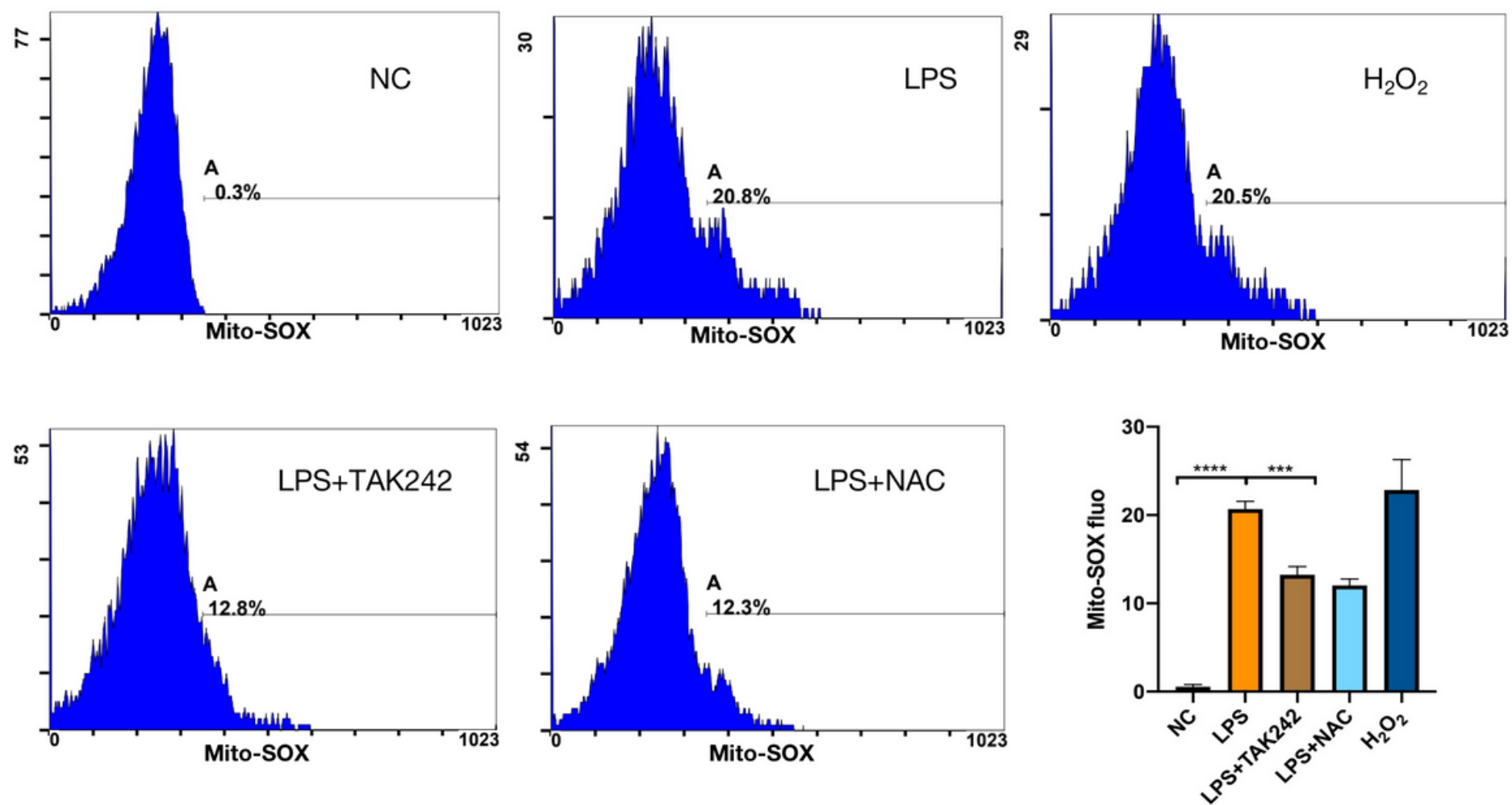

Figure 3

TAK242 decreased mtROS production induced by LPS in platelets. mtROS production was assayed by flow cytometry, $n=3$ in each group. The histogram displays the MFI of mtROS. Measurement data are represented as mean \pm SEM, ${ }^{\star \star *} \mathrm{p}<.001,{ }^{\star \star \star *} \mathrm{p}<.0001$. 

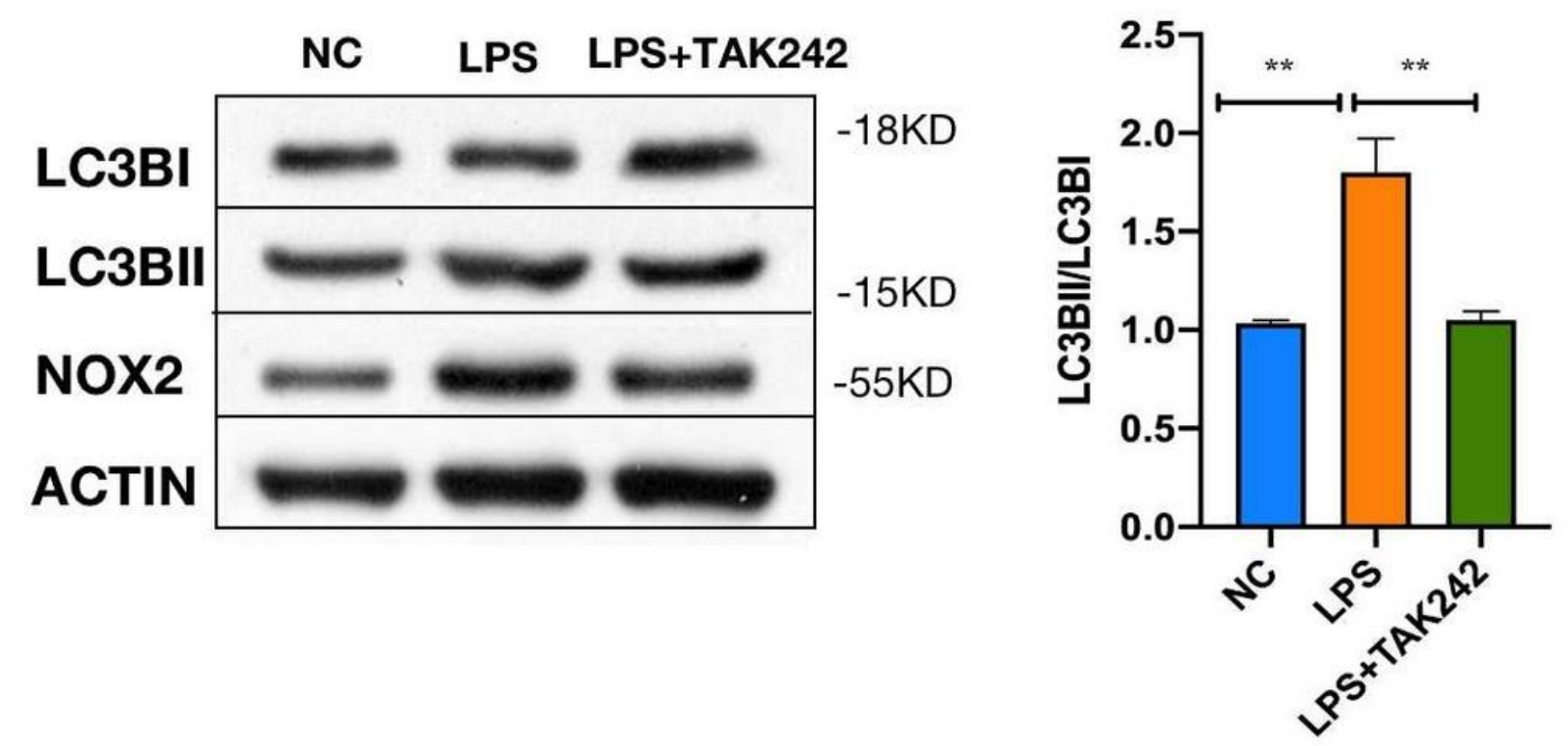

Figure 4

TAK242 decreased autophagy and NOX2 expression induced by LPS in platelets. The expression of LC3B and NOX2 were detected by Western blotting. Representative blotting images (left) and densitometric analysis (right) are shown, $\mathrm{n}=3$ in each group. Measurement data are represented as mean $\pm \mathrm{SEM}$, ${ }^{\star} \mathrm{p}<$ $.05,{ }^{\star *} \mathrm{p}<.01$. 